\title{
CAMBIOS EN LAS TRANSICIONES EDUCACIÓN-TRABAJO EGRESADOS DEL SECUNDARIO DEL GRAN BUENOS AIRES
}

\section{Agustina María Corica y Analía Elizabeth Otero}

\section{Resumen}

El objetivo de este texto es brindar evidencia de las transiciones de un segmento de la juventud en Argentina: los egresados 2011 de la educación secundaria. La idea vertebral es que durante los primeros años de egreso las transiciones van cambiando según las posibilidades concretas de combinar o excluir educación y trabajo, configurando trayectorias de vida diversas. Este análisis expone ejercicios metodológicos cuantitativos sobre los recorridos realizados y destaca las movilidades entre la continuidad educativa posegreso y las combinaciones del grupo que estudia y trabaja.

Palabras clave: juventud, educación, trayectorias, transiciones.

\section{Abstract}

Changes in education-work transitions. Graduated from the Gran Buenos Aires secondary

The objective of the text is to provide evidence of the transitions of a youth segment in Argentina: 2011 graduates of secondary education. The vertebral idea is that during the first years of graduation the transitions are changing according to the concrete possibilities of combining or excluding education from work, configuring diverse life paths. This analysis exposes quantitative methodological exercises on the routes carried out highlighting mobility between post-graduate educational continuity and combinations of the group that studies and works.

Keywords: youth, education, trajectories, transitions.

\footnotetext{
Agustina María Corica: Doctora en Ciencias Sociales por la Facultad de Ciencias Sociales, Universidad de Buenos Aires (UBA). Magíster en Diseño y Gestión en Políticas y Programas Sociales por la Facultad Latinoamericana de Ciencias Sociales (FLACSO-Argentina). Coordinadora académica del Programa de Investigaciones de Juventud de la FLACSO-Argentina. Investigadora adjunta del Consejo Nacional de Investigaciones Científicas y Técnicas (CONICET).

ORCID iD: 0000-0002-4096-6841

Email: agustinacorica@gmail.com

Analía Elizabeth Otero: Doctora en Ciencias Sociales y magíster en Diseño y Gestión de Programas y Políticas Sociales por la Facultad Latinoamericana de Ciencias Sociales (FLACSO). Investigadora adjunta del Consejo Nacional de Investigaciones Científicas y Técnicas (CONICET), investigadora del Instituto de Investigaciones Sociales de América Latina e investigadora principal del Programa Juventud de la FLACSO-Argentina. ORCID iD: 0000-0001-6774-1434

Email: aotero14@gmail.com
}

Recibido: 25 de julio de 2019

Aprobado: 16 de octubre de 2019 


\section{Introducción ${ }^{1}$}

Los estudios sobre la transición educación-trabajo constituyen una modalidad particular de abordaje e intentan dar cuenta de las tendencias al cambio y a la reproducción social a través del estudio de situaciones sociohistóricas concretas. El proceso de transición entre la educación y el mundo del trabajo tiene especificidades en las distintas etapas del ciclo vital; en ellas las circunstancias políticas, económicas y sociales imprimen efectos diversos. Asimismo, los hitos y rituales sociales que enmarcan las transiciones van cambiando de sentido y dirección a la luz de las tendencias generales vigentes en cada momento histórico, expresando inclusive movimientos inesperados o contradictorios. Además, pareciera que los cambios más importantes se dan en los primeros años de las transiciones y son diferenciados entre grupos específicos, por ejemplo, aquellos que lograron finalizar la escuela secundaria y aquellos que no. Uno de los hitos y eventos que estarían indicando cambios significativos en la vida de los jóvenes es cuando empiezan a trabajar (la edad mínima legal de ingreso al trabajo es a partir de los 16 años, con la excepción de las tareas productivas agrarias, en las que se permite el ingreso desde los 14 años) y otro cuando terminan la secundaria (la edad teórica de finalización del nivel medio de enseñanza es los 18 años). ${ }^{2}$

Inmersos en estas reflexiones, una idea central aquí es que durante los primeros años de egreso estos procesos de transición son inestables y van cambiando de rumbo, así como de dirección, según las posibilidades concretas

1 Este texto retoma parte de los hallazgos expuestos en la ponencia de las mismas autoras, titulada La transición educación-trabajo: los primeros años de las trayectorias educativas y laborales de jóvenes argentinos, presentada en el XXXI Congreso ALAS Uruguay: Las encrucijadas de América Latina. La sociología en tiempos de cambio, Montevideo, 3 al 8 de diciembre de 2017.

2 En Argentina se han establecido leyes de carácter integral, como la 26.061, de 2005, de Protección Integral de los Derechos de las Niñas, Niños y Adolescentes; la 24.650, de 1996, sobre la Edad Mínima de Admisión al Empleo; la 25.255, de 2000, sobre las Peores Formas de Trabajo Infantil, y la 26.390, de 2008, sobre la Prohibición del Trabajo Infantil y Protección del Trabajo Adolescente, en la cual se eleva la edad mínima de admisión al empleo a 16 años. A las anteriores normas se suman otras, sectoriales, que se aplican al universo adolescente, como la Ley 26.606 de Educación Nacional o la 25.673 de Salud Sexual y Procreación Responsable. La Ley Nacional de Educación extiende la obligatoriedad de la educación secundaria hasta la finalización del ciclo a la edad de 18 años. Se complementa con la legislación reciente en materia de pasantías educativas (Ley 26.427, de 2008, y Decreto 1.374, de 2011), que regula las prácticas laborales de estudiantes. 
de combinar, complementar o excluir educación y trabajo, configurando trayectorias de vida diversas. Pero, además, las características de estos procesos de transición pueden estar sufriendo variaciones sustantivas frente a las transformaciones en el sistema educativo y en el mercado de trabajo dadas en el marco del capitalismo global.

En este sentido, autores como Furlong y Cartmel ya expresaban cómo el modelo lineal y moderno de transición mediante el cual se explicaban sociológicamente las biografías empezó a dar signos de fatiga, en la medida en que la crisis industrial de los años ochenta se acentuó, haciéndose cada vez más complejas las transiciones desde la educación al trabajo y diversificándose cada vez más los itinerarios biográficos (Furlong y Cartmel, 2007, pp. 34-35). En línea con esta idea es que los trabajos como los de Du BoisReymond y López Blasco (2004) han denominado a este proceso de pérdida de linealidad y homogeneidad como diversificación, desestandarización o individualización de las transiciones. Autores como Giddens (1984), Beck (1992) y Bauman (2003) abordan la idea central de que los individuos, ante el gran abanico de posibilidades entre las que - parecen poder- optar, son impulsados a asumir constantemente de forma individual riesgos y consecuencias que antes eran asumidos de forma colectiva.

Por lo tanto, una premisa central en este trabajo es atender la multiplicación, singularización, desincronización y fragmentación de las transiciones juveniles contemporáneas (Walther et al., 2002) mediante el concepto de trayectorias. Así, autores como Feixa (2006) o Machado País (2007), entre otros, han abordado conceptos como el de trayectoria yo-yo para incorporar una de las novedades: la reversibilidad — con idas y vueltas - de las trayectorias biográficas que describen cada vez más jóvenes. En los contextos italiano y español, autores como Gentile (2010) han contribuido a este tipo de perspectivas desde el concepto de boomerang kids, para estudiar cómo un número creciente de jóvenes retornan al hogar familiar tras una experiencia de emancipación.

Frente al concepto de transición, el de trayectoria permite abordar con mayor precisión las discontinuidades de los recorridos biográficos y la creciente diversidad de contradicciones estructurales. Autores como Walther y Plug (2006, p. 77) o, más recientemente, Santamaría (2012) son ejemplos de este tipo de abordajes al dar importancia a la subjetividad y a las diferentes racionalidades de los jóvenes en la interpretación de sus biografías.

Partiendo de tales consignas, el objetivo general de este texto es aportar evidencia de las transiciones entre la educación y el trabajo para un segmento particular de la juventud en Argentina. Se trata de una cohorte de egresados en 2011 de la educación secundaria. Más precisamente, se analizan las características de dichos procesos con base en los datos de una investigación que metodológicamente se apoya en un seguimiento longitudinal, mediante el cual se pudo observar la diversidad de recorridos desde el año de finalización de la escuela secundaria hasta cinco años a posteriori. 
Para ello, en un primer apartado se reseñan notas sobre el proyecto de investigación. Seguidamente, se plantean algunos ejes centrales del debate teórico sobre la temática y se recogen perspectivas de estudios actuales que disertan sobre las características, heterogeneidades y variables incidentes en los procesos de transición. Se retoman estudios que aportan datos empíricos recientes, sobre todo del contexto argentino, a los que se suma el análisis elaborado a partir de investigaciones desarrolladas hace más de una década por nuestro equipo de trabajo. Los apartados siguientes se dedican a profundizar en el análisis elaborado. Luego, a modo de cierre, se presenta una reflexión acerca de lo planteado y se sugieren algunos interrogantes pendientes de profundización a partir de nuevas críticas que alimenten la discusión.

\section{Notas metodológicas sobre la investigación}

Este trabajo se ha desarrollado en el marco del proyecto "Itinerarios posibles o itinerarios probables: Un estudio sobre trayectorias educativas y laborales de jóvenes de distintos sectores sociales, egresados de la escuela media en Argentina", ${ }^{3}$ cuyo objetivo general ha sido indagar en las trayectorias educativas y ocupacionales de los egresados de la educación secundaria de una cohorte de 1999 y otra de 2011 que ingresan al mercado de trabajo en distintos contextos económicos, abarcando a jóvenes que viven en la ciudad y la provincia de Buenos Aires.

Esta propuesta retoma los hallazgos de los proyectos: "La inserción ocupacional de los egresados de la escuela media"4 y "La inserción ocupacional de los egresados de la escuela media: 10 años después". ${ }^{5}$ Tanto la investigación antecedente como el proyecto actual se enmarcan en los estudios longitudinales y utilizan la técnica de seguimiento de egresados, basada en un modelo follow-up studies,${ }^{6}$ que aplica distintas herramientas cuantitativas (encuesta 2011 preegreso y encuestas 2012 y 2016 posegreso) y

3 Proyecto: "Itinerarios posibles o itinerarios probables: Un estudio sobre trayectorias educativas y laborales de jóvenes de distintos sectores sociales, egresados de la escuela media en Argentina" (2014-2016), FLACSO, sede Argentina; Agencia Nacional de Promoción Científica y Tecnológica (PICT/2013 -0522).

4 Proyecto: "La inserción ocupacional de los egresados de la escuela media" (1998-2003), FLACSO, sede Argentina; Agencia Nacional de Promoción Científica y Tecnológica (PICT/98 04-04129); CONICET (PIP 0146/98).

5 Proyecto: "La inserción ocupacional de los egresados de la escuela media: 10 años después" (2008-2013), FLACSO, sede Argentina; Agencia Nacional de Promoción Científica y Tecnológica (PICT/2008-0531).

6 El método de follow-up se aplica en estudios que interrogan a estudiantes desde los años anteriores a su egreso y los encuestan en años posteriores. Tienen la potencialidad de poder reconstruir la trayectoria educativa de manera más acabada, por la cercanía con el ámbito escolar. Finalmente, la expresión estudios de seguimiento (follow-up) apunta a que los encuestados son antiguos estudiantes a quienes se ha aplicado un cuestionario después de titularse. 
cualitativas (30 entrevistas semiestructuradas realizadas en 2016). De modo que la estrategia metodológica implementada fue la triangulación de datos, en la que se combinaron distintos métodos de relevamiento de información (Cowman, 1993). Este tipo de abordaje metodológico contribuye a profundizar en el conocimiento de las trayectorias juveniles, en cuanto a la transición de la educación al trabajo, y permite una aproximación más comprensiva de los fenómenos implicados, ya que posibilita abarcar diferentes dimensiones, además de cotejar las situaciones en que se encuentran los jóvenes en distintos períodos temporales.

El seguimiento se realizó en la cohorte 2011 a jóvenes que asistían al último año de la escuela secundaria (en algunos casos fue el $5 .^{\circ}$, en otros, el $6 .^{\circ}$ año de la educación secundaria, dependiendo la orientación educativa), en distintas modalidades educativas, y posteriormente se realizó un relevamiento cuantitativo (telefónico) durante los primeros años de su inserción laboral (2012 y 2016), de modo de explorar acerca de las nuevas características de las inserciones possecundaria.

La composición de la muestra de estudiantes se elaboró a partir de la selección de una muestra de establecimientos educativos de carácter intencional y no probabilístico. La selección de establecimientos educativos se realizó a partir de los criterios clásicos para el análisis de la segmentación educativa. Siguiendo la tradición de los estudios del campo de la sociología de la educación, se distinguieron tres segmentos (bajo, medio, alto) y se tomaron en cuenta los siguientes indicadores: a) infraestructura escolar; b) titulación de los docentes; c) características socioeconómicas de la población que asiste. La muestra de escuelas quedó definida de la siguiente manera: 11 escuelas de modalidad bachiller, 5 de modalidad técnica, 2 de modalidad agraria y 1 modalidad artística. El número de escuelas que formaron parte de la muestra fue de 19.

En cuanto a la cantidad de alumnos encuestados, en la cohorte 2011 se encuestó a 538 alumnos: un $40 \%$ de sector bajo, un 38\% de sector medio y un $20 \%$ de sector alto. Las escuelas que integraron la muestra están localizadas en las ciudades de Buenos Aires y La Plata, el conurbano bonaerense y el interior de la provincia de Buenos Aires. Al año de haber egresado, se volvió a contactar a los alumnos y se logró encuestar a 385. Durante los años siguientes, se alcanzó a contactar a 301 egresados de la cohorte 2011 y se obtuvo un panel de casi el $60 \%$ de la muestra total. Cabe señalar que para la reconstrucción de los paneles se consideraron muestras homogéneas y representativas de la composición diferencial por sector social, geográfico y de género, para que no existiera sesgo estadístico.

Por lo tanto, la información compilada por el equipo de investigación permite analizar las principales transformaciones en la transición educacióntrabajo de los jóvenes en la ciudad y la provincia de Buenos Aires desde principios del siglo XXI. La cohorte analizada (2011) agrupa a jóvenes que 
nacieron entre 1993 y 1994, que, en su mayoría, llegaron a egresar a los 18 años de edad de la escuela secundaria, en un contexto caracterizado por la disponibilidad de oportunidades laborales y protección social.

En los apartados siguientes se profundiza en el análisis cuantitativo de los itinerarios de la cohorte 2011, vinculados fundamentalmente con la continuidad educativa posegreso, así como de las combinaciones y características del grupo que estudia y trabaja. También se consideran las distintas trayectorias y situaciones posegreso de los jóvenes del estudio, utilizando la información relevada en los tres tiempos del seguimiento de egresados, a modo de construir y conocer las configuraciones de las transiciones educación-trabajo en estos primeros años con el objetivo de mostrar las particularidades de esta cohorte.

\section{Procesos de transición ¿en vías de cambio?}

Como es conocido, en el campo de los estudios sobre juventud, en las últimas décadas han cobrado cierta prioridad aquellos referidos a lo laboral, entendidos en vinculación con las transformaciones macroestructurales en términos de los cambios económicos, históricos y sociales más amplios del actual contexto. Temáticas recurrentes de debate han sido y siguen siendo en nuestros días las variaciones y la heterogeneidad de las trayectorias juveniles, así como las transiciones entre la educación y el mundo del trabajo.

Los procesos de transición de los jóvenes y sus características vienen siendo estudiados en distintos países latinoamericanos. En las investigaciones recientes se destaca el carácter heterogéneo y situado de las juventudes, las distintas formas de vivenciarlas, las peculiaridades que revisten en el capitalismo global y las principales problemáticas que atraviesan la condición juvenil (Gotero y Weller, 2015).

Partiendo del debate sobre el vínculo entre la educación y el mundo del trabajo, e inscribiéndolo en las discusiones acerca de las caracterizaciones respecto a la transición educación-trabajo, como advertimos, el objetivo de este texto es brindar evidencia de las transiciones de un segmento de la juventud en Argentina: los egresados 2011 de la educación secundaria. El argumento central es que durante los primeros años de egreso las transiciones van cambiando según las posibilidades concretas de combinar o excluir educación y trabajo, configurando trayectorias de vida diversas. Este análisis, en particular, expone ejercicios metodológicos cuantitativos sobre los recorridos realizados y destaca las movilidades entre la continuidad educativa posegreso y las combinaciones del grupo que estudia y trabaja.

En las investigaciones de campo sobre las juventudes existe relativo consenso en torno a que los jóvenes, como grupo social, experimentan transiciones que dejan de ser lineales (educación para el empleo) y estandarizadas, como se entendían antaño. Hoy muchos jóvenes no solo 
combinan el estudio con el trabajo, sino que lo alternan: estudian, trabajan un tiempo y vuelven a estudiar, entre otras variantes (Saravi, 2015). En este sentido, se subraya el carácter heterogéneo que revisten las transiciones. Al mismo tiempo, existe disparidad de situaciones aun entre el grupo que comparte la realización de una única/excluyente actividad o entre quienes no realizan ninguna de ellas o entre aquellos que combinan en distintas variantes.

Respecto al mundo del trabajo y la inserción laboral, la incertidumbre es un rasgo observado por la mayoría de los estudios sobre las juventudes del presente siglo. Por cierto, es como un síntoma de un cambio generacional (aunque no resulta plenamente novedoso, ya que desde la década del setenta se produce un quiebre en el pleno empleo) que logra profundidad a fines de los noventa y 2000. Este es un aspecto sobre el carácter contemporáneo de las mismas transiciones.

De acuerdo con algunos autores, el tiempo entre que se deja de estudiar y se ingresa en el mercado laboral es una etapa clave, ya que marca la vida adulta. Los desafíos que demanda una inserción laboral adecuada son múltiples y en los diagnósticos sobre la transición los balances muestran que actualmente "este período es relativamente largo en la región latinoamericana". Además, resulta ser entendido como un período no solo largo, sino también cargado de incertidumbre. De allí que avanzar sobre la problemática significa contribuir a dilatar los obstáculos o a crear las condiciones para compensar más rápido, con la generación de experiencias relevantes, un modo de nutrir trayectorias laborales para que adquieran una orientación ascendente, sobre todo para aquellos que parten de situaciones más desventajosas, es decir, los grupos más vulnerables (Gotero y Weller, 2015, p. 68). ${ }^{7}$

\section{Tiempo joven}

El camino de la transición puede ser considerado en sí mismo un tramo con desafíos y múltiples pruebas u obstáculos a superar por los jóvenes. Las respuestas a estas pruebas pueden dar pistas para avanzar en la compresión de las trayectorias laborales/profesionales/vitales. Es decir, pueden constituirse en conocimientos útiles para guiar las intervenciones públicas en acciones estratégicas, fundamentalmente contribuyendo a acompañar los tránsitos y generar mayores condiciones de bienestar para el conjunto.

En el marco local, este problema es analizado desde diferentes perspectivas y dimensiones. El grueso de los estudios se centra en las trayectorias laborales de los sujetos jóvenes, considerando grupos con distintas caracterizaciones (por ejemplo, jóvenes de determinado sector

7 Recuérdese que el rasgo históricamente característico de la región es la profunda desigualdad que afecta con creces al sector poblacional joven, como muestran estudios recientes (CEPAL, 2018). 
social de pertenencia, en distintos ámbitos institucionales, trayectorias educativas, etcétera). Parte de las investigaciones que indagan con mayor especificidad en las transiciones entre educación y trabajo comprenden el trayecto posterior al nivel medio y la inserción laboral/continuidad educativa u otras ocupaciones.

También vale decir que en las últimas décadas en Argentina se produce una expansión institucional universitaria, especialmente en el territorio del Gran Buenos Aires. Este fue el escenario protagónico de la ampliación institucional, con la creación de 16 universidades $^{8}$ que se distribuyeron en los 24 municipios que integran un territorio caracterizado por concentrar la mayor parte de la población que vive en Argentina. ${ }^{9}$ Ello se sumó a las modificaciones legislativas realizadas a partir de la Ley de Educación Nacional (2006) y las políticas educativas implementadas en las últimas décadas, tanto en el nivel medio como en el superior, que delinearon trayectorias formativas más democráticas (Corica y Otero, 2017). En este sentido, Marquina y Chiroleau (2015) sostienen que las políticas inclusivas y democratizadoras en la educación superior en Argentina han tomado crucial relevancia en la política pública del último período, ampliando los programas de financiamiento estudiantil.

En este marco, en los análisis realizados para la presente investigación, se observa la propensión a la continuidad de los estudios superiores entre los jóvenes de la cohorte 2011 (Corica y Otero, 2015, 2018), lo que parecería indicar que se registran cada vez más trayectorias educativas extendidas.

Es más, siguiendo datos censales (Polo, 2016; Otero y Corica, 2015), en el largo plazo, una mirada en conjunto del sector poblacional joven indica que la tendencia es que cada vez más los jóvenes del país apelan a la combinatoria de estudio con trabajo. Investigaciones locales recientes indican que las continuidades educativas en combinatoria con las actividades laborales resultan una alternativa frecuente entre jóvenes de sectores medios y altos, en muchos casos ligadas al área de formación profesional a futuro o motivadas, entre otros, por la adquisición de experiencias acumulables (Busso y Pérez, 2015; Otero y Corica, 2017).

8 En el período 2004-2015 y luego de una de las crisis socioeconómicas más profundas de la Argentina, nuevas orientaciones en la política educativa dan marco a la creación de 16 universidades nacionales (Marquina y Chiroleau, 2015). Nuevamente el territorio privilegiado fue el Gran Buenos Aires, con la creación, por ley, de 8 universidades nacionales: Universidad Nacional de Avellaneda (2009), Universidad Nacional de Moreno (2009), Universidad Nacional Arturo Jauretche (2009), Universidad Nacional de José C. Paz (2009), Universidad Nacional del Oeste (2010), Universidad Nacional de Hurlingham (2014), Universidad Scalabrini Ortiz (San Isidro, 2015), Universidad Almirante Brown (2015).

9 Según el censo de 2001, la población de este distrito era de 8.684.437, y ascendió a 9.916 .715 de habitantes en 2010, según el último censo. Considerando que el país tiene 40 millones de habitantes, el Gran Buenos Aires es de gran interés para analizar tendencias. 
Más allá de esta mirada general, la combinación tiene sus particularidades según el sector social de proveniencia. La intensidad con que se realiza la actividad laboral y sus condiciones contextuales resultan menos favorables para los sectores bajos, lo que muestra los efectos de la dinámica estructural: los patrones de precariedad y vulnerabilidad para este sector no parecen revertirse pese a la baja del desempleo o las tendencias coyunturales de crecimiento (Otero y Corica, 2015).

Cabe aclarar aquí que en los primeros años posegreso del secundario las experiencias laborales que tienen los egresados participantes en la investigación son, en su mayoría, precarias. Ahora bien, los datos relevados dan cuenta de que la diferencia entre jóvenes de distintos sectores sociales está, sobre todo, en el tipo de tarea que realizan o en la rama de actividad de los trabajos. La posibilidad de vincular esa experiencia con la carrera educativa es lo que hace la diferencia en sus trayectorias (Corica y Otero, 2018).

Con estos indicios es que, a partir del análisis de los datos relevados en campo, se consideró en primer plano la inestabilidad que caracteriza a las actividades de los primeros años a posteriori de la finalización de la educación secundaria. Partiendo de allí se elaboró un conjunto de herramientas para mirar más de cerca el proceso y sus alcances. En función de ello y entendiendo la inestabilidad como el carácter transversal en el marco del cual se configuran las transiciones educación y trabajo, también nos preguntamos si se dan y cómo se expresan las desigualdades en función del sector social y el género entre los jóvenes de la muestra.

\section{Las actividades entre los jóvenes egresados de la cohorte 2011}

En anteriores análisis parciales desarrollados en el marco de esta misma investigación y, por tanto, mediante la utilización de la base de datos aquí retomada, se ha arribado a una serie de hallazgos que dan cuenta de aspectos que hacen a la caracterización y el perfil de los recorridos a lo largo de todo el período de seguimiento. Sintéticamente, estos hallazgos se reducen a cuatro rasgos centrales: a) se alarga la obtención del título secundario a un año de posegreso; b) más jóvenes continúan estudiando carreras universitarias; c) la actividad laboral es complementaria, no excluyente, a la continuidad educativa; y d) la dedicación horaria al trabajo es diferencial según el sector social de los jóvenes y según si estudian o no.

Con ese telón de fondo, la idea vertebral es, como se mencionó, que durante los primeros años de egreso las transiciones van cambiando según las posibilidades concretas de combinar o excluir educación y trabajo, configurando trayectorias de vida diversas. Para profundizar el análisis de la dinámica de las actividades de educación y de trabajo que realizaron los 
jóvenes en estos cinco años posegreso, a continuación se avanza en ver las continuidades, combinaciones, alternancias, movilidades, reversibilidades e inestabilidades en los recorridos.

\section{Primeros años de egreso}

En este primer apartado analítico se presenta la distribución de las actividades educación-trabajo realizadas por los jóvenes en los distintos momentos de aplicación de encuestas durante el seguimiento. ${ }^{10}$ En el tiempo I, es decir, en el último año de la escuela secundaria, casi todos los jóvenes estudiaban solamente $(81,8 \%)$; solo un $18,1 \%$ estudiaba y trabajaba al mismo tiempo. $\mathrm{Al}$ año de haber egresado, es decir, en el tiempo II, la situación cambia: la cantidad de jóvenes que estudian se reduce a menos de la mitad (47,4\%), el porcentaje de jóvenes que realizan ambas actividades (estudiar y trabajar) se incrementa apenas a un 20,7\%, pero comienzan a aparecer otras situaciones, que son las de aquellos que pasan a trabajar como actividad principal y única $(21,5 \%)$, es decir, que luego del egreso dejan de estudiar. A su vez, surge un grupo, que representa a un $10,4 \%$, que no estudia ni trabaja.

Continuando con el registro de las situaciones en los distintos tiempos del seguimiento de egresados, la situación a los cinco años de egresar del secundario (tiempo III) vuelve a cambiar. Los jóvenes que se dedican a estudiar solamente siguen disminuyendo, esta vez a un $31,8 \%$. Por el contrario, el grupo que combina ambas actividades se incrementa más que en la instancia anterior y alcanza un $40,8 \%$, y lo mismo ocurre con los jóvenes que trabajan solamente, que aumentan casi $1 \%$ la proporción de la instancia anterior, es decir, 22,8\%. En cambio, el grupo de jóvenes que al año de haber egresado no estudiaba ni trabajaba disminuye a casi la mitad: $4,5 \%$. Es decir, durante los primeros años posteriores a haber finalizado la escuela secundaria los jóvenes suelen cambiar las actividades que realizan e incluso pasar de la inactividad a la actividad (esto ocurre tanto en los casos de quienes no trabajaban como en los de quienes no realizaban ninguna de las dos actividades).

10 Para realizar el ejercicio metodológico se decidió categorizar las distintas tomas de datos: tiempo I (año 2011), para la primera encuesta realizada a los estudiantes de la muestra que asistían al último año de la escuela secundaria; tiempo II (año 2012), para la encuesta telefónica aplicada a la misma población a un año del egreso; y tiempo III (año 2016), para la encuesta telefónica aplicada a los cinco años de haber egresado. 


\section{Gráfico 1. Distribución porcentual de las actividades realizadas por jóvenes de la cohorte 2011, según toma de datos $[2011,2012,2015]$}

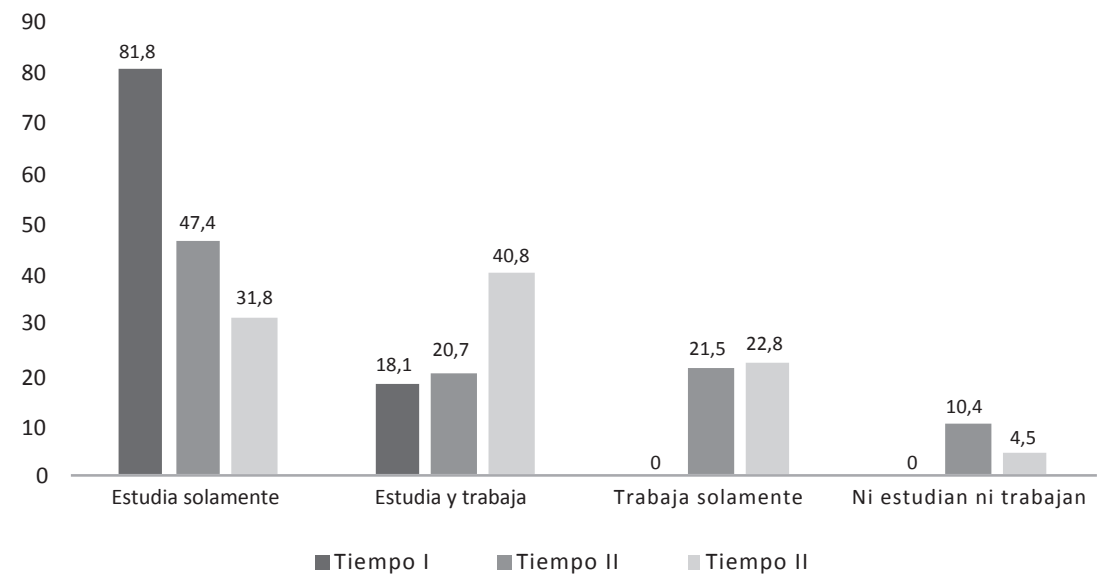

Fuente: Elaboración propia. Proyecto "Itinerarios posibles o itinerarios probables: Un estudio sobre trayectorias educativas y laborales de jóvenes de distintos sectores sociales, egresados de la escuela media en Argentina", FLACSO-USAL.

De modo que los datos relevados en el seguimiento de egresados dan cuenta de la variabilidad, movilidad y reversibilidad de las situaciones por las que pueden pasar los jóvenes en estos primeros años de la transición educación-trabajo a partir de las trayectorias recorridas a lo largo de los cinco años posteriores al egreso, así como de las tendencias generales según el momento y el ciclo de la transición en la que se encuentren.

Pero los datos también posibilitan profundizar en las tendencias y situaciones registradas a partir de la reconstrucción de las trayectorias que fue realizando cada uno de los egresados encuestados. Esta reconstrucción tendrá por objetivo constatar las primeras tendencias registradas en las distintas tomas o corroborar otros aspectos y elementos que influyen o definen estos cambios, como el género, el sector social de la escuela a la que asistieron o la posibilidad de revisar qué tipos de actividades, tanto educativas como laborales, han cambiado o revertido la situación original. Para ello, en el apartado siguiente se realiza un ejercicio metodológico y se definen aspectos centrales para la construcción de las trayectorias de los egresados 2011 de la escuela secundaria abarcados por el estudio.

\section{Hacia la construcción de una tipología de las trayectorias}

Según lo desarrollado en el apartado anterior y como forma de profundizar en el análisis de los recorridos transitados por el conjunto de jóvenes involucrados en el estudio, aquí se presenta una propuesta de síntesis y caracterización de distintos perfiles de trayectorias. 
La propuesta se basa en una nueva reagrupación cuantitativa que retoma las actividades realizadas por cada una de los jóvenes en las distintas tomas de datos, es decir, el tiempo I (en 2011, cuando aún transitaban el último año de la escuela secundaria), el tiempo II (en 2012, al año del egreso) y el tiempo III (en 2016, pasados cinco años del egreso).

El ejercicio metodológico permite captar la dinámica de las actividades realizadas durante los tres tiempos y, a partir de ello, caracterizar y describir la continuidad o las variaciones de las actividades de estudio y de trabajo, su combinatoria o la ausencia de ambas.

En función de lo anterior, se entiende posible observar el predominio de la continuidad o el cambio. Para ello se consideró una agrupación de las trayectorias a partir de las actividades vinculadas a lo educativo y lo laboral que los jóvenes realizaron en los distintos tiempos. En este sentido, se establecieron las siguientes definiciones:

\section{a. Descripción de trayectorias según temporalidad:}

1. Continuas: son aquellas trayectorias que implican la realización de la/s misma/s actividad/es en los tres momentos de toma de datos, es decir: los jóvenes estudian solamente o trabajan solamente o estudian y trabajan.

2. Discontinuas: son aquellas trayectorias que incluyen la realización en alguno de los tres tiempos de una sola de las actividades (los jóvenes estudian o trabajan) y en otros de los tiempos presentan ambas actividades combinadas. Se excluyen de esta categoría los casos de los que no estudian ni trabajan en alguno de los tiempos.

3. Alternas: son todas aquellas trayectorias que alternan las actividades entre los tres tiempos, es decir: en un momento los jóvenes estudian solamente, en otro momento trabajan solamente y en otro estudian solamente. En esta categoría se excluyen los casos que combinan estudio y trabajo en alguno de los tres tiempos.

4. SINSIN: son todas aquellas trayectorias en las que en alguno de los dos momentos posteriores al egreso (tiempo II o tiempo III) los jóvenes estaban sin estudiar y sin trabajar.

Si se observa el Cuadro 1, es notorio que el mayor porcentaje de la muestra se encuentra en las trayectorias que combinan el estudio con el trabajo $(53,35 \%)$, mientras que quienes realizan alguna de estas actividades (estudiar o trabajar luego del egreso) de forma continua son un $28,7 \%$. Por otro lado, se registra que durante los primeros cinco años posegreso un $12,8 \%$ de los jóvenes tienen trayectorias que en alguno de los momentos de toma de datos no se encontraban realizando actividades educativas ni laborales (SINSIN). A su vez, aparecen, de manera novedosa, las trayectorias en las que se alternan estudios y trabajo, que representan solo un $5,2 \%$ de los jóvenes. 
Por lo tanto, en este primer ejercicio de reconstrucción de las actividades educativas y laborales que realizan los jóvenes posegreso, las trayectorias más frecuentes son las discontinuas ${ }^{11}$ (más del $50 \%$ ), les siguen las continuas (casi un 30\%) y en menor medida las SINSIN (más de un 10\%) y alterna (cerca de un 5\%).

\section{Cuadro 1. Distribución de frecuencias y porcentual de jóvenes de la cohorte 2011, según trayectorias realizadas por temporalidad}

\begin{tabular}{ccc}
\hline Trayectorias & Frecuencia & Porcentaje \\
\hline Continua & 83 & 28,7 \\
\hline Discontinua & 154 & 53,3 \\
\hline SINSIN & 37 & 12,8 \\
\hline Alterna & 15 & 5,2 \\
\hline Total & 289 & 100,0 \\
\hline
\end{tabular}

Fuente: Elaboración propia. Proyecto "Itinerarios posibles o itinerarios probables: Un estudio sobre trayectorias educativas y laborales de jóvenes de distintos sectores sociales, egresados de la escuela media en Argentina", FLACSO-USAL.

A su vez, en estas trayectorias reconstruidas se consideró el sector social de la escuela de la cual provienen los jóvenes y si eran hombres o mujeres. De allí, surgen diferencias que resaltan la desigualdad de los itinerarios. En esta dirección, la mayoría de las trayectorias SINSIN se verifican entre los jóvenes provenientes de las escuelas de sectores bajos. En cambio, las trayectorias con continuidad se encuentran entre los jóvenes de los sectores medios y altos. En cuanto a la distinción según el género, la distribución de las proporciones entre las trayectorias continua y discontinua son bastante semejantes, aunque en las continuas predominan las mujeres $(32,2 \%)$ frente a los varones $(25 \%)$ y en las discontinuas predominan los varones $(56,9 \%)$ frente a las mujeres $(49,7 \%)$. En cambio, en las trayectorias SINSIN, predominan las mujeres (14\%), aunque la proporción de varones es de un $11 \%$ - hay solo una pequeña diferencia de $3 \%$ - y en las trayectorias que alternan predominan los varones $(6,9 \%)$ por sobre las mujeres $(3,5 \%)$.

11 Estas trayectorias discontinuas son presentadas por jóvenes que combinan discontinuamente actividades educativas con actividades laborales. Son los casos que, en un primer tiempo, hacían ambas actividades, en un segundo tiempo solo una actividad y en el tercer tiempo vuelven hacer ambas actividades de forma combinada, por ejemplo. 


\section{Cuadro 2. Trayectorias por temporalidad para la cohorte 2011 según sector social de la escuela y sexo}

\begin{tabular}{ccccccc}
\hline \multirow{2}{*}{ Trayectorias } & \multicolumn{7}{c}{ Sector social de la escuela } & \multicolumn{2}{c}{ Sexo } & \multirow{2}{*}{ Total } \\
\cline { 2 - 6 } & Bajo & Medio & Alto & Femenino & Masculino & \\
\hline Continua & 21,9 & $\mathbf{3 1 , 4}$ & $\mathbf{3 2 , 0}$ & 32,2 & 25,0 & 28,7 \\
\hline Discontinua & $\mathbf{4 0 , 6}$ & $\mathbf{5 7 , 9}$ & $\mathbf{6 1 , 3}$ & 49,7 & 56,9 & 53,3 \\
\hline Alternas & 6,6 & 6,6 & 1,3 & 3,5 & 6,9 & 5,2 \\
\hline SINSIN & $\mathbf{3 0 , 8}$ & 4,1 & 5,3 & 14,7 & 11,1 & 12,8 \\
\hline Total & 100,0 & 100,0 & 100,0 & 100,0 & 100,0 & 100,0 \\
\hline
\end{tabular}

Fuente: Elaboración propia. Proyecto "Itinerarios posibles o itinerarios probables: Un estudio sobre trayectorias educativas y laborales de jóvenes de distintos sectores sociales, egresados de la escuela media en Argentina", FLACSO-USAL.

Como segundo ejercicio metodológico y analítico se consideró el agrupamiento anterior, pero pensando en la direccionalidad de las trayectorias que los jóvenes habían desarrollado a lo largo de estos cinco años posegreso. En este sentido, se realizaron las agrupaciones calificando las actividades (educativas y laborales) según la direccionalidad del trayecto realizado en los itinerarios educativos y los laborales a lo largo de los tres tiempos, donde se encontraron líneas rectas, circulantes, entrecruzadas y yuxtapuestas. Por eso, se procedió al reagrupamiento de las trayectorias en una nueva formulación categorial basada en graficar estos trayectos. Para ello se consideraron y adecuaron a los fines del estudio las definiciones de la RAE de las siguientes palabras: 1) lineal, perteneciente o relativo a la línea; en este estudio, hace referencia a la representación de un trayecto de forma alargada y estrecha, como una línea, y que se repite progresivamente y manteniendo la misma proporción; 2) vacilante, que se mueve indeterminadamente, titubea, está poco firme en su estado; en el estudio, se aplica a trayectos que tienden a moverse de un lado a otro por falta de estabilidad o equilibrio y que muestran vacilación o indecisión; y 3) acoplada, cuando una pieza está acoplada, significa que fue ajustada al sitio en el que debe ser colocada o agrupada con otras de manera tal que su funcionamiento combinado produce el resultado conveniente; en el estudio los trayectos identificados con esta categoría presentan tramos que se integran y funcionan armónicamente.

En este sentido, se resignificaron los trayectos e itinerarios registrados a partir de pensar en un mapa de movimientos en cuanto a inicio y terminalidad de las actividades educativas y laborales que realizan los egresados incluidos en la presente investigación. Para ello, las categorías lineal, vacilante y acoplada dan cuenta de las trayectorias, que van en distintas direcciones (vacilantes), que marcan trazos que se unen o se integran (acopladas) o en una 
dirección, como las trayectorias lineales. Así, se avanzó hacia la construcción de una tipología de trayectorias según direccionalidad. ${ }^{12}$

\section{b. Descripción de trayectorias según direccionalidad:}

1. Lineales: son todas aquellas trayectorias de los jóvenes que, en los tiempos I, II y III, estudian solamente; también aquellas de los que en los tiempos II y III trabajan solamente o no estudian ni trabajan en ambos tiempos. Es decir, aquellas trayectorias de jóvenes que, posegreso, realizan la misma actividad (se excluyen las de aquellos que realizan en el tiempo I ambas actividades).

2. Vacilantes: son todas aquellas trayectorias que involucran la alternancia de las actividades, en un tiempo los jóvenes estudian solamente y en otro trabajan solamente, y aquellas de los jóvenes que no estudian ni trabajan de forma discontinua.

3. Acopladas: son todas aquellas trayectorias que presentan la combinación de ambas actividades en alguno de los tres tiempos (se incluyen las de aquellos que realizan en el tiempo I ambas actividades).

Según los datos procesados, las trayectorias que predominan entre los jóvenes egresados de la muestra son: la trayectoria acoplada, con el 56,4\%, a la que le siguen las trayectorias lineales, con un $26,3 \%$, y las vacilantes, con $17,3 \%$. Entre las vacilantes encontramos la mayoría de casos de jóvenes que no estudian y no trabajan, y en menor medida a quienes alternan el estudio un año y otro año el trabajo, sin combinar ni estabilizar a lo largo del tiempo las actividades que realizan. En cambio, en las trayectorias lineales predominan los casos de quienes estudian solamente y entre las trayectorias acopladas los que combinan ambas actividades (estudiar y trabajar) de forma discontinua (ver Anexo, Cuadro A1).

12 Rememorando las distintas tipologías propuestas por Casal (trayectorias de aproximación sucesiva) y Machado País (trayectorias yo-yo) es que se pensó en esta nueva categorización. Como trayectorias de aproximación sucesiva Joaquim Casal identifica itinerarios de jóvenes que apuntan hacia una inserción con éxito que les demanda toma de decisiones e itinerarios de formación prolongados, pero también ciertas demoras o ajustes a las situaciones de estudio o trabajo y, finalmente, atrasos en el mismo proceso de emancipación familiar por razones económicas o de estrategia. El término es muy descriptivo de una forma basada en el ensayoerror y en la moratoria de decisiones complejas (elección de carrera, vida en pareja, créditos hipotecarios, etcétera). Por su parte, Machado País, por ejemplo, llama yo-yo a la trayectoria que va y vuelve de una condición a otra: el estudiante que comienza a trabajar y luego vuelve a ser estudiante, o el dependiente que pasa a la independencia y luego vuelve a la dependencia. Ahora bien, la idea de pensar estas nuevas categorías mediante el ejercicio metodológico es poner el foco en la variabilidad de rumbos o cambios de trayectorias que cuestionan el carácter lineal y reversible de los procesos de transición. 


\section{Cuadro 3. Distribución de frecuencias y porcentual de jóvenes de la cohorte 2011, según trayectorias realizadas por direccionalidad}

\begin{tabular}{ccc}
\hline Trayectorias & Cantidad & Porcentaje \\
\hline Lineales & 76,0 & 26,3 \\
\hline Vacilantes & 50,0 & 17,3 \\
\hline Acopladas & 163,0 & 56,4 \\
\hline Total & 289,0 & 100,0 \\
\hline
\end{tabular}

Fuente: Elaboración propia. Proyecto "Itinerarios posibles o itinerarios probables: Un estudio sobre trayectorias educativas y laborales de jóvenes de distintos sectores sociales, egresados de la escuela media en Argentina, FLACSO-USAL.

A su vez, las trayectorias según direccionalidad son distintas de acuerdo con el sector social de la escuela. Entre los jóvenes de los sectores bajos predominan las trayectorias vacilantes. En cambio, en los sectores medios y altos predominan las trayectorias acopladas. Mientras que las trayectorias lineales son, en su mayoría, de jóvenes pertenecientes a los sectores medios. Cabe señalar que no existen grandes diferencias entre hombres y mujeres según esta tipología de trayectorias.

Por lo tanto, las trayectorias posegreso que se fueron reconstruyendo a lo largo de los ejercicios metodológicos resaltan los aspectos y características diferentes y diferenciales según la temporalidad y la direccionalidad, así como las actividades que los jóvenes fueron realizando. Se destaca que hay trayectorias más inestables (las vacilantes) y más estables (las acopladas y lineales). Pero, a su vez y considerando los distintos agrupamientos realizados, las trayectorias más inestables presentan situaciones más complejas de revertir: son las que presentan mayoritariamente ausencia de actividad educativa a lo largo del análisis.

\section{Entre estabilidad y cambios}

Ahora bien, en una nueva etapa analítica hemos dado prioridad a la variable temporal, a partir de incorporar cuán móvil o no ha sido la distribución de las actividades realizadas por los jóvenes de la muestra al promediar el recorrido del seguimiento que hemos instrumentado mediante la presente investigación longitudinal. A modo de distinguir ciertas características que refieren más a las novedades-discontinuidades o, por el contrario, a la continuidad de modos de transición educación-trabajo, hemos reforzado en la variable temporal caracterizando las trayectorias a partir de su mayor o menor propensión a la movilidad. La descripción de esta tipología de trayectorias se detalla a continuación. 


\section{c. Descripción de trayectorias según movilidad:}

1. + Móviles: reúne las trayectorias de aquellos que, en los distintos tiempos, han cambiado las actividades que desarrolla.

2. - Móviles: reúne las trayectorias de aquellos que, en los tres tiempos, especialmente entre los tiempos II y III, no han cambiado de actividad.

Según el Cuadro 4, la síntesis establece la prevalencia de un mayor porcentaje de movilidad entre los recorridos de los jóvenes de la muestra, aunque la diferencia entre ambos tipos de trayectorias es de un $10 \%, 45,3 \%$ y $54,7 \%$ respectivamente.

\section{Cuadro 4. Trayectorias por movilidad según recorridos realizados en los cinco años posegreso. Cohorte 2011}

\begin{tabular}{ccc}
\hline Trayectorias & Cantidad & Porcentaje \\
\hline - Móviles & 131 & 45,3 \\
\hline + Móviles & 158 & 54,7 \\
\hline Total & 289 & 100 \\
\hline
\end{tabular}

Fuente: Elaboración propia. Proyecto "Itinerarios posibles o itinerarios probables: Un estudio sobre trayectorias educativas y laborales de jóvenes de distintos sectores sociales, egresados de la escuela media en Argentina", FLACSO-USAL.

Entre las trayectorias más móviles predominan las mujeres $(51,9 \%)$ y los jóvenes de los sectores medios (41\%) y bajos (33,3\%). Por el contrario, en las trayectorias menos móviles se destacan apenas los varones $(52,7 \%)$ por sobre las mujeres $(47,3 \%)$ y los jóvenes de los sectores medios $(43,5 \%)$ (ver Anexo, Cuadros A2 y A3).

Contrario a lo que se hubiera pensado, a partir de las tendencias generales sobre la mayor reversibilidad y los cambios en las trayectorias de los jóvenes, nos encontramos con que, al considerar el carácter de más o menos móvil, resultan de este ejercicio - y para esta muestra - dos grupos claros: el grupo de jóvenes que se encuentran en la trayectoria menos móvil se caracteriza por incluir a quienes en ambas tomas de datos del estudio longitudinal se encontraban estudiando solamente, es decir, son estudiantes y jóvenes de sectores medios $\mathrm{y}$ altos y son, en menor medida, también aquellos que trabajan solamente posegreso en los tiempos II y III, así como los que combinan en ambos tiempos ambas actividades; en cambio, el grupo cuyas trayectorias son más móviles se caracteriza por estar compuesto por jóvenes que son ambivalentes en sus actividades y son mayoritariamente de sectores bajos y medios: en una toma de datos combinan el estudio con el trabajo, en otra solo trabajan o solo estudian, o en alguna de esas tomas no realizan ninguna de las dos actividades. 
Por último, y a partir del ejercicio metodológico realizado, nos preguntamos sobre los factores y efectos de las temporalidades, la movilidad y las direccionalidades de las trayectorias y cuánto de estas variables puede tener un elemento explicativo en las trayectorias de los jóvenes en los procesos de transición educación-trabajo. Uno de los cuestionamientos de fondo en cuanto a la movilidad de las trayectorias de los jóvenes en estos primeros años de la transición es cuánto de estas experiencias está vinculado con la reversibilidad o no de situaciones precarias o en riesgo o de mayor vulnerabilidad que se perpetúan o predicen situaciones a futuro.

Debatir sobre conceptos como transición, trayectorias, reversibilidad y linealidad es uno de los objetivos finales de este artículo. El ejercicio metodológico cuantitativo longitudinal realizado puede abrir puntas para pensar la importancia o no de los primeros años de la transición o las formas en que se dan estos procesos, considerando que durante los primeros años de egreso las transiciones van cambiando según las posibilidades concretas de combinar o excluir la educación del trabajo, configurando trayectorias de vida diversas.

\section{A modo de cierre}

Según distintas investigaciones recientes, el pasaje de la juventud a la edad adulta se ha ido alargando en el tiempo, volviéndose su delimitación una línea borrosa. En este marco, los procesos de transición entre educación y trabajo tienen que repensarse con nuevas dimensiones y fenómenos que resultan de un progresivo proceso de desinstitucionalización y desritualización de este pasaje. En esta dirección es que en el presente artículo se abordó mediante un estudio longitudinal el análisis de estos procesos en los primeros años posegreso de la escuela secundaria de un grupo de jóvenes que viven en la ciudad y la provincia de Buenos Aires.

Este período del ciclo biográfico se ha universalizado y uniformizado con relación a los sectores sociales y el género de las personas jóvenes, especialmente por el alargamiento del proceso formativo que caracteriza a esta cohorte. Pero detrás de esta uniformidad surgen, a partir del ejercicio metodológico, aparentes fenómenos complejos, procesuales y graduales. Es decir, encontramos distintas trayectorias que se presentan más o menos estables y otras con cambios de rumbos que, a lo largo de los cinco años posegreso, se complejizan, algunas se convierten en situaciones vulnerables y otras logran trayectos más exitosos.

La adquisición de experiencias puede tener un peso importante en la acumulación de aprendizajes calificados. Como contracara, puede estar indicando, desde el inicio, condiciones desiguales que podrían tender a perpetuarse a futuro y a afectar el desarrollo en distintos planos vitales. De aquí surgen dos interrogantes de trasfondo a partir del ejercicio metodológico: 
por un lado, cuánto pesan las experiencias diferenciales de los primeros tramos y, por otro, cuánto dicen sobre las futuras posiciones en el mercado de trabajo.

En lo que atañe a lo laboral, el aporte de material empírico sobre los primeros años de la transición es relevante - aunque no predictivo- en el mapa de las tendencias, obstáculos y respuestas generadas por los jóvenes ante las situaciones que atraviesan. Los primeros años posegreso constituyen un modo de incursionar en la etapa inicial, frecuentemente de búsqueda de inserción laboral-primer empleo. No obstante, no puede obviarse que la dinámica inestable y las reversibilidades, alternancias, etcétera, en cuanto a las actividades que realizan los jóvenes, vuelven necesaria la búsqueda de acercamientos longitudinales y diacrónicos más que fotografías a punto fijo. ${ }^{13}$

Por otro lado, la evidencia empírica analizada da cuenta de que las trayectorias son heterogéneas en su configuración. Los datos arrojan que un poco más de la mitad de las trayectorias son móviles, sin embargo, el resto de ellas sigue teniendo estabilidad en las actividades posegreso. Este dato confirma que la idea de linealidad que caracterizaba la transición educación-trabajo en décadas anteriores se debilita. Las trayectorias también se registran como discontinuas y acopladas en su mayoría. Es decir, dejan de estar estandarizadas e institucionalizadas en cuanto al orden en el cual se realizan las actividades educativas y laborales a lo largo del tiempo, así como en cuanto a los cambios de instituciones donde se desarrollan ambas actividades.

Por lo tanto, el ejercicio de reconstrucción de trayectos educativos y laborales de jóvenes egresados del nivel medio arroja indicios de que los procesos de transición tienden a ser cada vez más diversos y distintos. Esto se ve especialmente en las trayectorias configuradas según la temporalidad, donde la discontinuidad, continuidad, alternancia e inactividad educativas y laborales resultan ser diferentes entre mujeres y varones y entre los distintos grupos sociales. También da cuenta de que la transición educación-trabajo es un proceso que se da a lo largo del tiempo y que implica cambios de rumbos que, en algunos grupos sociales, parecieran ser reversibles. Sin embargo, del ejercicio también resulta que, a mayor movilidad en los primeros años, mayor probabilidad de trayectorias erráticas o vulnerables. Según los datos procesados en estas situaciones, en su mayoría, se trata de las mujeres de sectores bajos que presentan en su transición mayores cambios de actividades educativas y laborales, inclusive alcanzando en algunos momentos la inactividad. Por otro lado, encontramos a jóvenes de los sectores medios y altos, varones, que continuaron estudios superiores y tienen experiencias laborales vinculadas con sus estudios, así como a mujeres de estos grupos sociales con trayectorias universitarias y con antecedentes laborales calificados.

13 Esto hace tomar en cuenta ciertos recaudos, ya que no son situaciones estáticas. Justamente, el rasgo característico es la inestabilidad. 
En este sentido, se observa que la distinción de trayectorias según las tipologías construidas invisibiliza muchas veces desigualdades educativas y laborales en cuanto a la diferenciación en términos de calidad y calificación de los trabajos que obtienen y en cuanto a los estudios possecundario que logran realizar (carreras universitarias, terciarias o de formación profesional). Por ejemplo, las mujeres de sectores medios y bajos que lograron continuar estudiando, en algunos casos, combinaron sus estudios con trabajos esporádicos y no calificados, y han cambiado de carrera, inclusive pasando de carreras universitarias a estudios terciarios. Presentan trayectorias discontinuas, vacilantes y más móviles. Por otro lado, podemos encontrar a varones de sectores medios que presentan trayectorias vacilantes y más móviles, pero que a lo largo de la transición van realizando, de forma gradual, su proceso de inserción laboral con experiencias laborales calificadas, revirtiendo situaciones complejas.

Las situaciones descriptas son algunas de las que integran la diversidad de situaciones que encontramos detrás de la clasificación en los distintos tipos de trayectorias utilizados en el ejercicio metodológico, con el objetivo de mostrar que se hacen necesarias, para pensar las trayectorias educativas y laborales juveniles, nuevas categorías que distingan las experiencias educativas y laborales por calidad, tipo o lugar, ya que las características de esas experiencias (universitarias o de formación profesional, trabajos de operarios o profesionalizantes) en los primeros años posegreso van a ser claves para revertir la desigualdad de origen.

\section{Referencias bibliográficas}

Bauman, Z. (2003). Comunidad. En busca de seguridad en un mundo hostil. Madrid: Siglo XXI.

Beck, U. (1992). From industrial society to the risk society: questions of survival, social structure and ecological enlightenment. Theory, Culture y Society, 9(1), pp. 97-123.

Busso, M. y P. Pérez (2015). Combinar trabajo y estudios superiores. ¿Un privilegio de jóvenes de sectores de altos ingresos? Revista Población y Sociedad, 22, pp. 5-29.

Casal, J.; M. García; R. Merino y M. Quesada (2006). Aportaciones teóricas y metodológicas a la sociología de la juventud desde la perspectiva de la transición. Papers, 79, pp. 21-48.

Corica, A. y A. Otero (2017). Después de estudiar, estudio... Experiencia de jóvenes egresados de la escuela media. Revista Población y Sociedad, 24(2), pp. 33-64. 
Corica, A. y A. Otero (2018). Transiciones juveniles: un análisis sobre el vínculo educación y trabajo de jóvenes egresados de la educación obligatoria argentina. Revista Última Década, 48, pp. 133-168.

CEPAL (Comisión Económica para América Latina y el Caribe) (2018). La ineficiencia de la desigualdad 2018. Santiago de Chile: CEPAL.

Cowman, S. (1993). Triangulation: a means of reconciliation in nursing research. Journal of Acvanced Nursing, 18, pp. 788-792.

Du Bois-Reymond, M. y A. López Blasco (2004). Transiciones tipo yo-yo y trayectorias fallidas: hacia las políticas integradas de transición para los jóvenes europeos. Revista de Estudios de Juventud, 65(04), pp. 11-29.

Feixa, C. (2006). Generación XX. Teorías sobre la juventud en la era contemporánea. Revista Latinoamericana de Ciencias Sociales, Niñez y Juventud, 4(2), pp. 21-45.

Furlong, A. y F. Cartmel (2007). Young people and social change. New York: McGraw Hill.

Giddens, A. (1984). The constitution of society: Outline of the theory of structuration. Cambridge: Polity Press.

Gotero, S. y J. Weller (2015). ¿Estudias o trabajas? El largo camino hacia la independencia económica de los jóvenes de América Latina. Serie Macroeconomía del Desarrollo, 169. Santiago de Chile: CEPAL.

OCDE/CEPAL/CAF (2016). Perspectivas económicas de América Latina 2017. Juventud, competencias y emprendimiento. París: OECD Publishing.

Machado País, J. (2007). Chollos, chapuzas, changas. Jóvenes, trabajo precario y futuro. Barcelona: Anthropo.

Otero, A. y A. Corica (2015). Perspectivas educativas y laborales de los jóvenes latinoamericanos: tendencias y desafíos. Revista Latinoamericana de Estudios Educativos, XLV(2), pp. 9-42.

Polo, R. (2016). ¿Cómo combinan los jóvenes asistencia a la escuela y trabajo? Evolución de las combinaciones posibles durante el periodo 1970-2010. Análisis de las diferencias según género y edad, y por regiones del país. Ponencia presentada en las IX Jornadas de Sociología de la UNLP. Facultad de Humanidades y Ciencias de la Educación de la Universidad Nacional de La Plata 5, 6 y 7 de diciembre de 2016. 
Santamaría, E. (2012). Trayectorias laborales en los márgenes del empleo: políticas, subjetividades y experiencias de jóvenes en la precariedad laboral. Vitoria-Gasteiz: Administración de la Comunidad Autónoma del País Vasco.

Saraví, G. A. (2015). Juventudes fragmentadas. Socialización, clase y cultura en la construcción de la desigualdad. México DF: Centro de Investigaciones y Estudios Superiores en Antropología Social y Facultad Latinoamericana de Ciencias Sociales-México.

Walther, A.; B. Stauber; A. Biggart; M. du Bois-Reymonds; A. Furlong; A. López Blasco; S. Mørch; J. M. Pais (eds.) (2002). Misleading Trajectories? Integration Policies and Young Adults in Europe. Opladen: Leske+Budrich.

Walther, A. y W. Plug (2006). Transitions from school to work in Europe: Destandardization and policy trends. New Directions for Child and Adolescent Development, 113, pp. 77-90.

\section{Contribución de autoría}

El trabajo fue realizado en un 50\% por Agustina María Corica y en un 50\% por Analía Elizabeth Otero. 
Anexo

Cuadro A1. Trayectorias por direccionalidad para la cohorte 2011, según actividades realizadas a lo largo del seguimiento

\begin{tabular}{clccc}
\hline \multirow{2}{*}{ Trayectorias } & \multicolumn{1}{c}{ Actividades } & Cantidad & Porcentaje* $^{*}$ \\
\hline \multirow{2}{*}{ Lineales } & Estudia solamente & 55 & 72,4 \\
\cline { 2 - 4 } & Trabaja solamente & 19 & 25,0 \\
\cline { 2 - 4 } & SINSIN continuo & 2 & 2,6 \\
\hline \multirow{2}{*}{ Vacilantes } & Estudia/Trabaja & 15 & 30,0 \\
\cline { 2 - 4 } & SINSIN discontinuos & 35 & 70,0 \\
\hline \multirow{3}{*}{ Acopladas } & Combinan-continuo & 9 & 5,5 \\
\cline { 2 - 4 } & Combinan continuo posegreso & 7 & 4,3 \\
\cline { 2 - 4 } & Combinan discontinuo & 147 & 90,2 \\
\hline
\end{tabular}

Fuente: Elaboración propia. Proyecto "Itinerarios posibles o itinerarios probables: Un estudio sobre trayectorias educativas y laborales de jóvenes de distintos sectores sociales, egresados de la escuela media en Argentina", FLACSO-USAL. * EI porcentaje se presenta por trayectoria.

\section{Cuadro A2. Trayectorias por temporalidad para la cohorte 2011, según sector social de la escuela y sexo}

\begin{tabular}{ccccccc}
\hline \multirow{2}{*}{ Trayectorias } & \multicolumn{7}{c}{ Sector social de la escuela } & \multicolumn{2}{c}{ Sexo } & \multirow{2}{*}{ Total } \\
\cline { 2 - 6 } & Bajo & Medio & Alto & Femenino & Masculino & \\
\hline Menos móviles & $29,8 \%$ & $\mathbf{4 3 , 5 \%}$ & $26,7 \%$ & $47,3 \%$ & $\mathbf{5 2 , 7 \%}$ & $100,0 \%$ \\
\hline Más móviles & $\mathbf{3 3 , 3 \%}$ & $41,0 \%$ & $25,6 \%$ & $\mathbf{5 1 , 9 \%}$ & $48,1 \%$ & $100,0 \%$ \\
\hline Total & $31,7 \%$ & $42,2 \%$ & $26,1 \%$ & $49,8 \%$ & $50,2 \%$ & $100,0 \%$ \\
\hline
\end{tabular}

Fuente: Elaboración propia. Proyecto "Itinerarios posibles o itinerarios probables: Un estudio sobre trayectorias educativas y laborales de jóvenes de distintos sectores sociales, egresados de la escuela media en Argentina", FLACSO-USAL.

\section{Cuadro A3. Trayectorias por temporalidad para la cohorte 2011, según sector social de la escuela y sexo}

\begin{tabular}{cccccc}
\hline \multirow{2}{*}{ Trayectorias } & \multicolumn{3}{c}{ Sector social de la escuela } & \multicolumn{2}{c}{ Sexo } \\
\cline { 2 - 6 } & Bajo & Medio & Alto & Femenino & Masculino \\
\hline Menos móviles & $42,9 \%$ & $\mathbf{4 7 , 1} \%$ & $\mathbf{4 6 , 7} \%$ & $43,4 \%$ & $47,9 \%$ \\
\hline Más móviles & $\mathbf{5 7 , 1} \%$ & $52,9 \%$ & $53,3 \%$ & $\mathbf{5 6 , 6} \%$ & $\mathbf{5 2 , 1 \%}$ \\
\hline Total & 100,0 & 100,0 & $100,0 \%$ & $100,0 \%$ & $100,0 \%$ \\
\hline
\end{tabular}

Fuente: Elaboración propia. Proyecto "Itinerarios posibles o itinerarios probables: Un estudio sobre trayectorias educativas y laborales de jóvenes de distintos sectores sociales, egresados de la escuela media en Argentina; FLACSO-USAL. 